\title{
Forecasting energy time series with profile neural networks
}

\author{
Benedikt Heidrich \\ Karlsruhe Institute of Technology \\ Institute for Automation and Applied \\ Informatics \\ benedikt.heidrich@kit.edu
}

\author{
Marian Turowski \\ Karlsruhe Institute of Technology \\ Institute for Automation and Applied \\ Informatics \\ marian.turowski@kit.edu
}

\author{
Nicole Ludwig \\ Karlsruhe Institute of Technology \\ Institute for Automation and Applied \\ Informatics \\ nicole.ludwig@kit.edu
}

\author{
Ralf Mikut \\ Karlsruhe Institute of Technology \\ Institute for Automation and Applied \\ Informatics \\ ralf.mikut@kit.edu
}

\author{
Veit Hagenmeyer \\ Karlsruhe Institute of Technology \\ Institute for Automation and Applied \\ Informatics \\ veit.hagenmeyer@kit.edu
}

\section{INTRODUCTION}

With more renewable energy sources in the electricity network, the supply becomes more volatile, and hence balancing the grid becomes more challenging. Network operators, consequently, rely heavily on demand forecasts for scheduling the power supply to match the demand. This need for demand forecasts has led to various forecasting approaches being introduced as presented in e. $\mathrm{g}$. $[1,6,8,9,22,23]$.

Traditionally, statistical forecasting models are used, as they are easy to interpret while having a great forecasting performance [9]. However, in many areas, neural networks and especially deep neural networks have shown great success in learning difficult dependencies and uncover hidden knowledge in large data sets [16]. This ability could be of great importance for energy time series forecasting as well, since the increasing use of smart meters allows to collect more and more time series data.

In fact, neural networks (NNs) are frequently used forecasting approaches [8] for energy time series. Additionally, stacking these NNs into deep neural networks [2] to form, for example, convolutional neural networks (CNNs) achieves promising results. CNNs outperform other machine learning approaches such as random forest [15] or support vector regression [3].

However, forecasting time series-especially time series that are periodic such as energy time series-is difficult for neural networks. They have been shown to struggle with learning periodicities and trends in view of having no inherent concept of time. Hence, they rarely outperform statistical forecasting approaches $[5,19]$. Nevertheless, in the latest M4 forecasting competition [20], the winning model combined statistical information about the seasonality with a deep learning approach, namely recurrent neural networks (RNNs) [25]. This strategy, in which the strength of the neural network and the strength of the statistical approaches are both leveraged, seems to be most promising for future forecasting models.

Thus, in the present paper, we introduce profile neural networks in line with the finding that deep learning can be improved with statistical information. This new architecture obtains explicit information about periodicities in the load time series and can then focus on learning what is hidden in the data and what simpler models might miss. In contrast to [25], we use the faster and more promising $\mathrm{CNN}$ in our model and give explicit periodicity information in the form of standard load profiles. Although using standard 


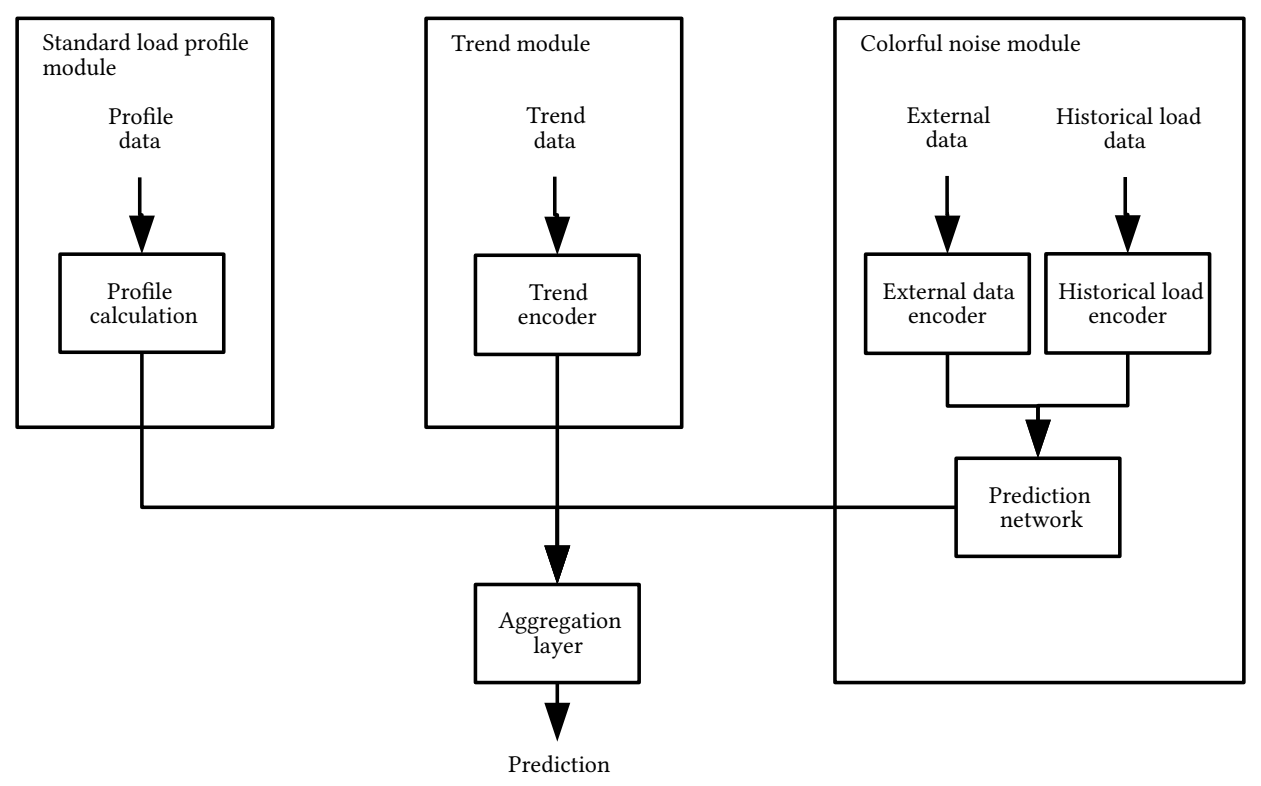

Figure 1: Overview of the profile neural network with its three modules, i. e. the standard load profile, trend, and colorful noise module, as well as the aggregation layer to form the final prediction.

load profiles is not a new concept in energy forecasting research [24] and practice [18] and neural networks have been applied to energy forecasting before, to the best of our knowledge no paper has combined standard load profiles and thus statistical information with CNNs before.

The remainder of the present paper is structured as follows. In Section 2, we introduce our profile neural network and explain convolutional neural networks as well as the architecture's three main modules, i. e. the standard load profile module, the trend module, and the colorful noise module. In Section 3, we then evaluate the architecture on building electricity load data from a university campus and compare the results of the architecture to benchmark models. We thereby investigate the performance for a one dayahead and a one week-ahead forecast on a building with strong seasonal variation and a building with weak seasonal variation. Finally, we discuss the architecture in Section 4 before concluding the paper in Section 5.

\section{PROFILE NEURAL NETWORKS}

In this section, we describe the newly proposed profile neural network (PNN) for energy load forecasting, which is publicly available on GitHub ${ }^{1}$. We base our architecture on the underlying idea that decomposing energy time series into a standard load profile, a trend, and a colorful noise module improves the forecasting. Before we introduce the modules and their aggregation in detail, we shortly describe CNNs, which we use throughout the architecture.

A convolutional neural network is a type of neural network that has become popular in sequence processing [10]. In contrast to simple neural networks, CNNs usually consist of three different types of layers: convolutional layers, pooling layers, and fully connected layers. In convolutional layers, filters are applied to extract

\footnotetext{
${ }^{1}$ https://github.com/benHeid/Profile-Neural-Network
}

features. These filters are composed of kernels that are learned and form feature maps. The feature maps are then activated through an activation function, typically a rectified linear unit. Pooling layers are mostly used to reduce the dimensionality of the network. For example, max pooling reduces a filter region to its maximum value. Lastly, fully connected layers are standard neural network layers that combine the features from the feature maps for forming the output and thus mostly used as the last layer. For a more detailed introduction to CNNs we refer to e. g. [10].

\subsection{Standard Load Profile Module}

The first module of the profile neural network is the standard load profile module. In the present paper, standard load profiles are typical electrical load patterns of a specific building as shown in Figure 2.

Since energy time series are periodic and NNs can hardly cope with these periodicities, NNs are not used for the profile calculation. Instead, the present paper uses the following algorithm to obtain a standard load profile. As input, the algorithm needs historical electrical load (input sequence), a window size, and range of time for which the profile should be calculated (target date range). The output is then a profile for the target date range (e. g. the profiles in Figure 2). To calculate the standard load profile, the algorithm performs the following four steps.

(1) Split the input sequence into days.

(2) Group these days according to the day of the week (e. g. Saturday). Note that public holidays are considered as Sundays.

(3) For each group $g$, calculate the moving average within the specified window using

$$
a_{g, t_{n}}= \begin{cases}\frac{1}{\left|L_{G, t_{n}, T}\right|} \sum_{l \in L_{G, t_{n}, T} l,} t_{n}-T \geq t_{0} \\ \frac{1}{\left|L_{G, t_{n}, t_{n}}\right|} \sum_{l \in L_{G, t_{n}, t_{n}} l,} l & \text { otherwise, }\end{cases}
$$




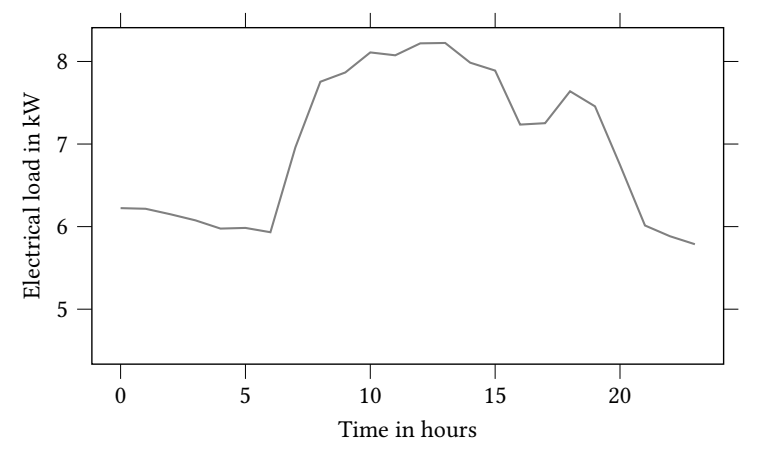

(a) Exemplary standard load profile for a 24 hours forecast horizon.

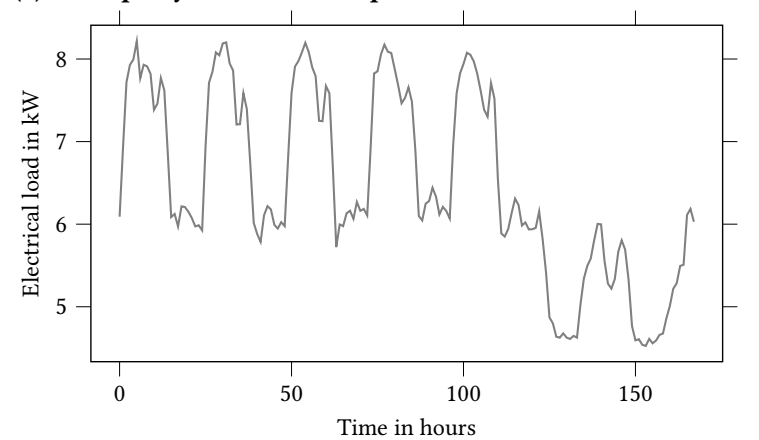

(b) Exemplary standard load profile for a 168 hours forecast horizon.

Figure 2: Exemplary standard load profiles for a 24 hours, i. e. one day, and a 168 hours, i. e. one week, forecast horizon.

where $L_{G, t_{n}, T}$ is the set of the vectors of the hourly electrical loads for each day in the group $G$ between $t_{n}-T$ and $t_{n}$. At this point, $t_{n}$ is the current time and $T$ is number of time steps representing the window size. The first element of the considered time series is the date $t_{0}$.

The second case in the formula is necessary because the number of available time steps is smaller than the window size at the beginning of the training sequence.

(4) For each date $t_{n}$ in the target date range, get the group $g$ of this date and take the corresponding $a_{g, t_{n}}$.

In general, other algorithms can also be used for calculating the standard load profile.

\subsection{Trend Module}

The second module of the profile neural network is concerned with the trend of the time series. In the present paper, a trend is any change in the level of the electrical load over the past few weeks. To obtain the trend data, we apply the algorithm by Lei et al. [17] to the difference between the original time series and the standard load profile. The algorithm extracts the past ten weeks of load information for the value to be predicted. Consequently, the trend data for the value

$$
q_{t}=\left[l_{t-p}, l_{t-2 * p}, \ldots, l_{t-10 * p}\right]
$$

is a vector of size ten, where $q_{t}$ is the trend, $l_{t}$ is the electrical load at time $t$, and $p$ is the length of one period (here 168 hours). We choose

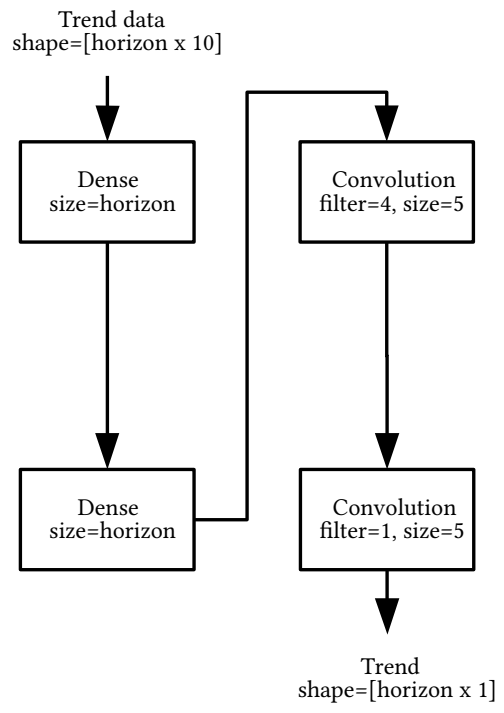

Figure 3: The trend encoder, responsible for encoding the trend information for each value to be predicted. It consists of two dense layers and two convolutional layers.

ten weeks because it is the proposed value by Lei et al. [17] and variations of this value show no improvement in the accuracy of the PNN. For more details see Lei et al. [17]. In contrast to Lei et al. [17], however, we predict the electrical load of the next horizon steps. Consequently, we extract the trend information for each value to be predicted. The result is trend data with the shape [horizon $\times 10$ ], which means that the trend data is a matrix with a length of horizon for the first dimension and a length of 10 for the second dimension.

This trend data is then used in a trend encoder as illustrated in Figure 3 to describe future trends. However, in contrast to Lei et al. [17], we use a trend encoder consisting of a CNN with two dense and two convolutional layers. In a first step, the two dense layers encode the trend for each value to be predicted locally, i. e. the dense layers process the trend data for each value to be predicted separately. In a second step, two convolutional layers combine the local trend information with the trend information of the neighbors. The key idea of this approach is to concentrate the trend information for each value to be predicted before combining it with the trend information of neighboring values to be predicted. Considering the trend information of the neighbors should ensure that adjacent values have similar trend information. This is important because we assume that adjacent values are influenced by the same trend. The trend encoder's output has the shape [horizon $\times 1$ ].

A benefit of the proposed trend encoder is that it retains the neighborhood. This means that the output of each layer of the trend encoder corresponds to one value to be predicted, thus making the network easier to understand because each neuron is matched to one value to be predicted.

\subsection{Colorful Noise Module}

The third module of the profile neural network is the colorful noise module. The colorful noise is the residual part of the time series that is neither learned through the trend encoder nor the application 


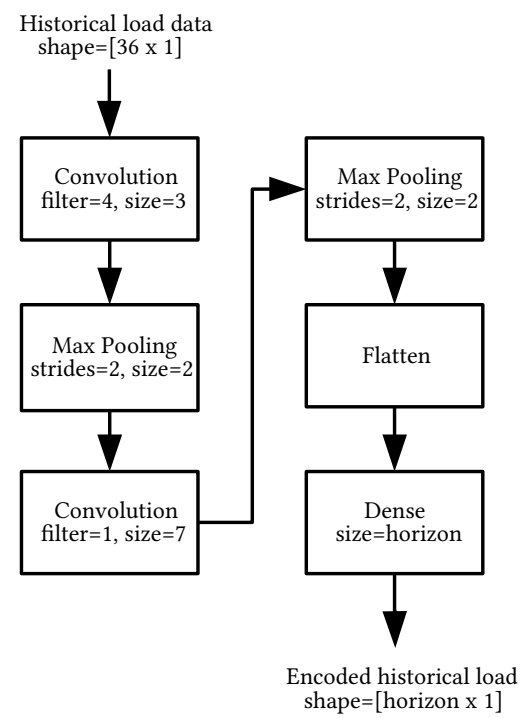

Figure 4: The historical load encoder, responsible for encoding the last $n$ values of the historical load. It consists of two convolutional layers and two pooling layers.

of a standard load profile. We use the term colorful noise because we assume that there is additional information in this noise that our model should discover. In order to predict the colorful noise, historical load data and external explanatory variables are used as inputs to the prediction network.

The historical load data consists of the last 36 hours of the electrical load, from which the standard load profile is subtracted. This data is fed into the historical load encoder illustrated in Figure 4. The encoder is a simple CNN, consisting of two convolutional layers and two pooling layers. The last layer of this encoder is a fully connected layer that is responsible for expanding the shape from $[36 \times 1]$ to [horizon $\times 1$ ]. This expansion is necessary to concatenate this encoder's output with the output from the external data encoder, which we describe in the following.

The external data encoder introduces external explanatory variables to the architecture. We use weather and date-related information as inputs to the encoder. The weather information includes humidity and temperature data, while the date-related information consists of the hour of the day, the day of the month, the month of the year, and dummy variables for each day of the week and one that specifies whether the day is a business day or not. Following [13], the hour of the day, the day of the month, and the month of the year are encoded as a sine function, ensuring that adjacent dates have adjacent numerical values. For example, January and December are adjacent months but a simple encoding would encode January with one and December with twelve, creating a numerical distance between the months. A sine encoding, however, avoids this behavior due to its periodicity. In the external encoder, we use two convolutional layers to keep the neighborhood information in the data (see Figure 5).

Finally, the prediction network illustrated in Figure 6 calculates the colorful noise. For this purpose, it takes the concatenated outputs of the external data encoder and historical load encoder as

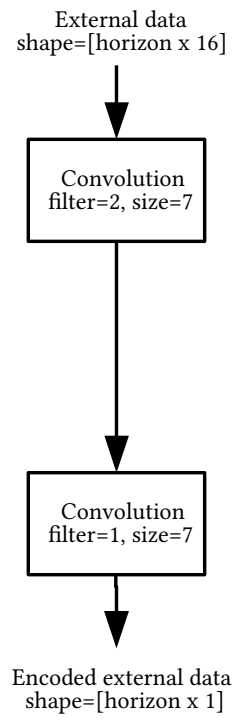

Figure 5: The external data encoder, responsible for encoding the weather- and date-related information. It consist of two convolutional layers.

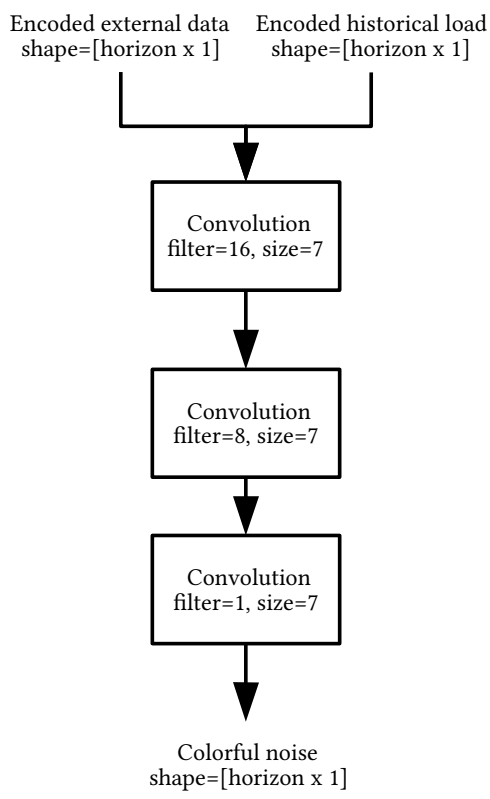

Figure 6: The prediction network, responsible for calculating the colorful noise using the encoded external data and encoded historical load. It consists of three convolutional layers.

inputs. The prediction network consists of three convolutional layers. As the colorful noise consists of only one value per target date, we reduce the filter size in each convolutional layer. 


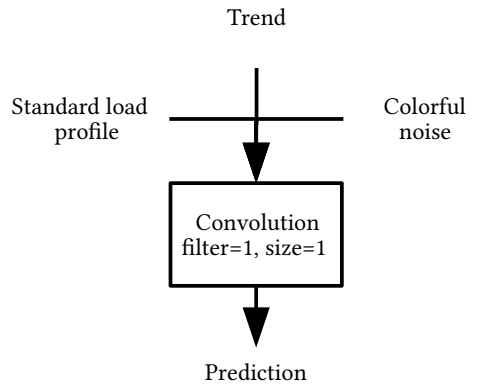

Figure 7: The aggregation layer, responsible for weighting the modules' output. It consists of a single convolutional layer.

\subsection{Aggregation layer}

In the last step, the three modules introduced above (standard load profile module, trend module, and colorful noise module) are aggregated to form the final output. For this purpose, different aggregation methods can be chosen, where the simplest one is an addition of the three modules. In the following, we describe our approach to this module aggregation, where we want to learn the importance of the individual modules.

In a first step, we concatenate the modules' output and then feed it into a convolutional layer (see Figure 7). This convolutional layer is responsible for weighting the importance of the three modules. We thus choose a kernel size and filter number of one. To ease the training process, we initialize the weights in the layer with $\frac{1}{3}$, thus an equal weighting of the modules. Furthermore, we remove the bias and use a linear activation function within the convolutional layer. The output of this layer is then the load forecast for the next horizon values.

\section{EVALUATION}

In this section, we evaluate the profile neural network (PNN) on building electricity load data and compare the performance to benchmark models. We thus introduce the used dataset and present the benchmark models before we describe the experimental setting together with the different evaluated PNN models and report the results.

\subsection{Dataset}

Our evaluation data comes from two datasets, which we introduce in the following together with their preprocessing steps.

The first dataset is the real-world electrical load data from buildings on a university campus. The dataset consists of smart meter readings that measure the electrical demand of the buildings every 15 minutes over more than ten years, i. e. January 1, 2006, to May 18,2016 . Due to measurement errors, we interpolate single missing and zero data points using a simple linear interpolation before we differentiate the time series to consider the change in the energy demand. As the time series still exhibits spikes, we only use data above zero and below the $99 \%$ quantile. Given this data, we select the electrical energy consumption of two different buildings on the campus for our analysis. As illustrated in Figure 8, the energy consumption of the first building shows weak seasonal variation while the energy consumption of the second building shows strong seasonal variation.

The second dataset comprises weather data by the German Meteorological Service, the Deutsche Wetterdienst (DWD) [7]. From the available station-based weather data, we select the data of the spatially closest station as the relevant subset. The dataset consists of the air temperature at two-meter above ground and the humidity for a period of more than ten years, i. e. November 1, 2008, to December 31, 2018. The measurements are available in an hourly resolution.

The performed preprocessing of both datasets consists of two steps. Firstly, we merge the two datasets. For this purpose, we crop both datasets such that both cover the same period, i. e. November 1, 2008, to May 18, 2016. Secondly, we adapt the temporal resolutions of both datasets to each other by using the mean of one hour to aggregate the electrical energy demand measurements to one hour.

\subsection{Benchmark Models}

In order to accurately compare the performance of our new architecture, we train seven benchmark models from the literature on our data. Four benchmark models are based on state-of-theart neural networks, and three are naive forecasting models. The naive forecasting models are used to establish a lower benchmark which should be outperformed in any case, while the state-of-theart neural network models should show the competitiveness of our PNNs.

The three naive forecasting benchmark models with increasing complexity are the periodic persistence forecast with a periodicity of one week, the profile forecast, and the linear regression model. The periodic persistence forecast takes, for each value to be predicted, the corresponding value of the last week as the prediction, i. e.

$$
\hat{y}_{t}=y_{t-168}
$$

where $y_{t}$ is the electrical load and $\hat{y}_{t}$ is the prediction of the electrical load at time $t$.

The profile forecast uses the corresponding value of the standard load profile as forecast such that

$$
\hat{y}_{t}=p_{t}
$$

where $p_{t}$ is the value of the standard load profile with a window size of one month at time $t$.

Lastly, the linear regression model uses the same external variables as the state-of-the-art neural network benchmarks and can thus be written as

$$
\hat{y}=c+\sum_{j} \beta_{j} \cdot W_{j}+\sum_{l} \gamma_{l} \cdot T_{l}+\sum_{k} \delta_{k} \cdot D_{k}+\varepsilon,
$$

where $c$ is a constant, $W_{j}$ represents all weather variables (in this case relative humidity and temperature), $T_{l}$ are all trend variables (in this case a linear, a quadratic, and a cubic trend), and $D_{k}$ are all dummies such as weekend dummies and dummies for the different days of the week. The error $\varepsilon$ is modeled with an autoregressive model. Note that we dropped the time indices from the equation for simplicity. The model, and thus $\hat{y}$, is trained once on the colorful noise, in the following called linear regression model (noise), 


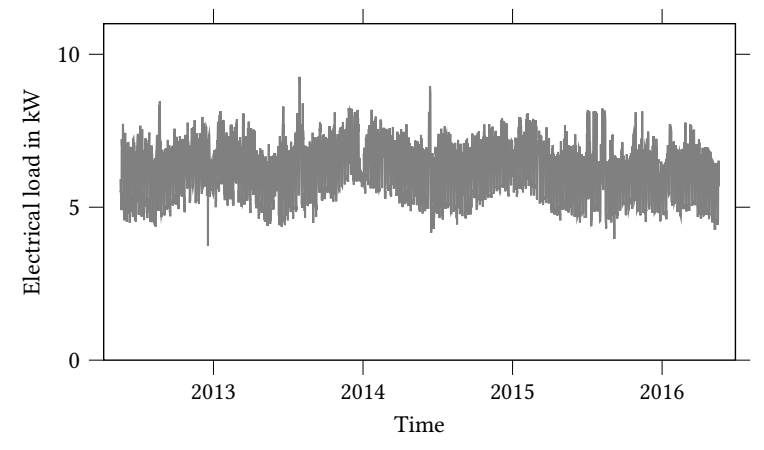

(a) The electrical load of the building with weak seasonal variation for the complete training and test set.

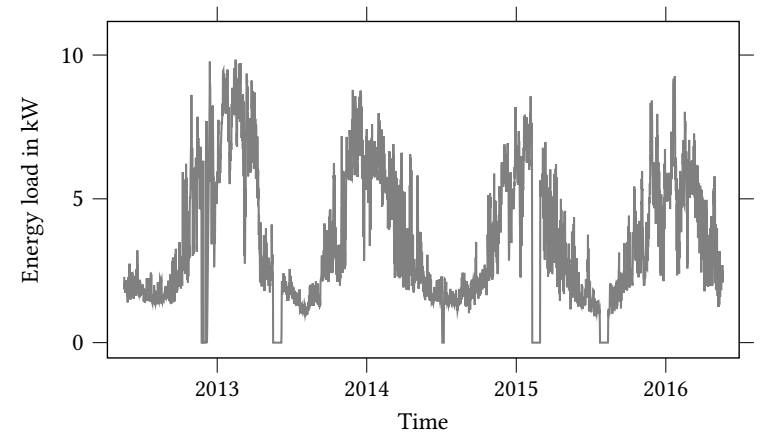

(c) The electrical load of the building with strong seasonal variation for the complete training and test set.

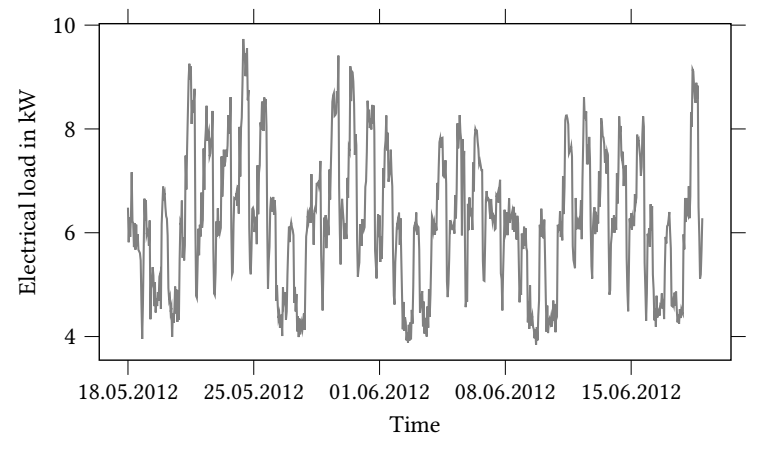

(b) The electrical load of the building with weak seasonal variation for one month.

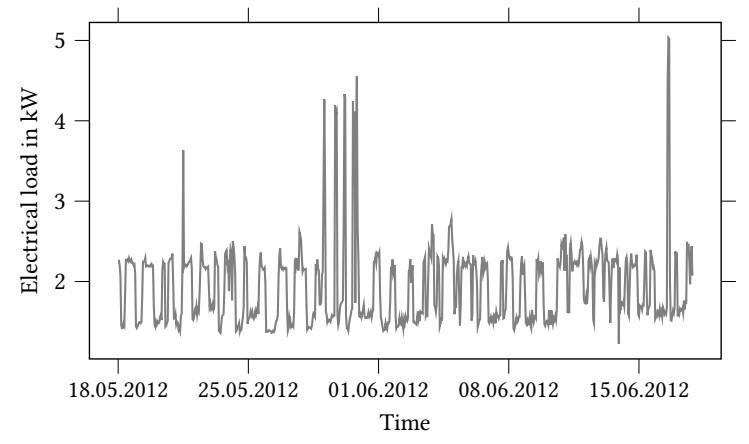

(d) The electrical load of the building with strong seasonal variation for one month.

Figure 8: Comparison of the electrical load time series of the two buildings considered over the complete training and test set, i. e. May 18, 2012, to May 18, 2016, as well as for one month.

and once on the original time series, in the following called linear regression model (original).

The first state-of-the-art neural network benchmark model is the RCFNet by [17]. The RCFNet is a CNN that extracts features like trend and periodicity information from the time series and uses the extracted information as additional input for the CNN. Since the original model only predicts the electrical demand of a single point of time in the future, we adapt its last layer such that it forecasts the electrical demand for the next 24 hours and 168 hours respectively. As our second state-of-the-art neural network benchmark model, we use the first CNN proposed by [3]. This network is a simple $\mathrm{CNN}$ that takes historical load and external data as inputs. In the following, we refer to this network as Amarasinghe network.

The two remaining state-of-the-art neural network benchmark models use Long-Short Term Memories (LSTMs). LSTMs are an improved variant of recurrent networks that allows the neurons to control the amount of old and new information used for forming the output [11]. The network of [21] stacks two LSTM layers for predicting the electrical load. The recurrent inception network by [13] is more complex as it combines LSTMs with an inception module. An inception module consists of multiple parallel convolutional layers with different kernel sizes. This structure allows the network to decide for itself which is the best kernel size for the specific task [26]. Furthermore, the recurrent inception network takes historical data together with context information (e. g. date information) as inputs.

\subsection{Experimental Setting}

This subsection introduces the evaluated PNN models and describes the experimental setting of this comparison.

We use three different PNN models for both forecast horizons, one day-ahead (24 hours) and one week-ahead (168 hours). These models differ only in the window size that is chosen to calculate the standard load profile. More specifically, we test window sizes of one month (PNN1), six months (PNN6), and twelve months (PNN12). Furthermore, to evaluate the standard load profiles, we also train our architecture without any standard load profile (PNN0).

For these four PNN models and the selected benchmark models, we compare the accuracy and training time needed. Since we randomly initialize the weights of the neural networks, we train and evaluate each model five times to achieve more robust results. Furthermore, for a better comparability of the results, we use the same hardware (see Table 1) for the calculations of each model, building, and forecast horizon.

For training and evaluating the models, we split the data of the two buildings. We use three years of data (May 18, 2012 until May 18, 2015) for the training and the remaining year (May 18, 2015 until May 18, 2016) for testing. 
Table 1: The hardware specification of the computer used for the evaluation.

$\begin{array}{ll}\text { Operating System } & \text { Windows 10 } \\ \text { Main Memory } & 16 \mathrm{~GB} \\ \text { CPU } & \text { Intel i7-8700K } 3.7 \mathrm{GHz} \\ \text { GPU } & \text { NVIDIA Quadro P2000 } \\ \text { Cuda Version } & 10 \\ \text { Graphic Memory } & 5 \mathrm{~GB} \\ \text { Keras Version } & 2.2 .4 \\ \text { Tensorflow Version } & 1.13 .1\end{array}$

For the training, we use batch sizes of 128 and the Adam optimizer [14]. Since we also measure the time needed for convergence, we set a maximum of 2000 epochs for the training. However, all models need less epochs, and we apply early stopping. As loss function for the PNN in the training process, we make use of the sum squared error (SSE)

$$
S S E=\sum_{t}\left(y_{t}-\hat{y}_{t}\right)^{2},
$$

where $y_{t}$ is the actually observed value and $\hat{y}_{t}$ is the predicted value. For the evaluation of the PNN models and benchmark models, we measure the accuracy with the root mean squared error (RMSE)

$$
R M S E=\sqrt{\frac{\sum_{t}\left(y_{t}-\hat{y}_{t}\right)^{2}}{n}},
$$

where $n$ is the number of predictions. In our case, we thus take the square root of the squared deviations between the value to be predicted and the predicted value divided by the number of days in the test set.

For a scale invariant comparison of our approach with the benchmarks, we also use the mean absolute scaled error (MASE) [12], i. e.

$$
\text { MASE }=\frac{\frac{1}{n} \sum_{t}\left|y_{t}-\hat{y}_{t}\right|}{\frac{1}{T} \sum_{t}\left|y_{t}-y_{t-p}\right|},
$$

as an additional metric. In this formula, $p$ is the period length (here 168 hours), $T$ is the length of the training set, and $n$ is the length of the test set.

\subsection{Results}

Given the evaluated PNN models, benchmark models, and our experimental setup introduced above, the results are explained in the following. In the first two parts, we compare the accuracy and the training time needed. Afterwards, we evaluate the interaction of accuracy and training time. Finally, we examine the decomposition provided by the PNN.

Accuracy. The accuracy-as defined in Equation (7) and Equation (8)of the models is presented in Figure 9a for the building with weak seasonal variation and in Figure $9 \mathrm{~b}$ for the building with strong seasonal variation. In each figure, the one day-ahead and the one week-ahead forecasts are shown. The bars indicate the root mean squared error (RMSE) on the left side, and the mean absolute scaled error (MASE) of the respective model on the right side with the grey error bars showing the worst and best RMSE and MASE respectively. Since the relative results are the same for both metrics, we focus on the RMSE in the following.
For the building with weak seasonal variation, our proposed PNN1 with a standard load profile outperforms the benchmark models for both forecast horizons. Compared to the best benchmark model (standard load profile based forecast), the average RMSE of PNN1 is $20 \%$ better for the one day-ahead and $12 \%$ better for the one week-ahead forecast. Note that the simple standard load profile forecast is better than the other neural network benchmark models. The worst result is obtained by the linear regression model on the original data for both forecast horizons. Furthermore, all PNNs with a standard load profile achieve a better accuracy than the PNN without a standard load profile (PNN0). Moreover, we observe that the PNN with smaller window sizes achieve better results than the PNN with larger window sizes. However, the accuracy of PNN12 and PNN6 is almost the same.

For the building with strong seasonal variation (Figure 9b), the best model is also the PNN1. For the one day-ahead forecast, PNN1 is $13 \%$ better than the recurrent inception networks in terms of the RMSE and $15 \%$ for the one week-ahead forecast. The model with the worst accuracy is the periodic persistence forecast. We also observe that the accuracy of the PNN6, the RCFNet, and the Amarasinghe network strongly varies for the two different forecast horizons while the PNN0 is distinctly worse than the other PNNs for both forecast horizons. An exception is the PNN6 for the one week-ahead forecast, whose accuracy is worse than that of PNN0.

Training time. Regarding the training time needed, we compare the PNN models to all neural network benchmark models.

For the building with weak seasonal variation, we observe that the PNN models need the shortest training time for the one dayahead forecast, followed by the Amarasinghe network and the LSTM network. However, for the one week-ahead forecast, the Amarasinghe network requires the shortest training time, followed by the stacked LSTM network and the PNN models. For both forecast horizons, the model with the longest training time is the recurrent inception network. Moreover, all models need more training time for the one week-ahead than for the one day-ahead forecast. Furthermore, for both forecast horizons, the results show that all PNN models need approximately the same training time.

Regarding the building with strong seasonal variation, an important observation is that the needed training time is shorter for each model than for the building with weak seasonal variation except for the recurrent inception network. For the one day-ahead forecast, the PNN models need the shortest training time, followed by the Amarasinghe network. However, for the one week-ahead forecast, the shortest training time is needed by the Amarasinghe network. The recurrent inception network requires the longest training time again.

Accuracy vs. training time. In the following, we put the obtained accuracy in relation to the training time needed using scatter plots (see Figure 10), where the average training time needed is plotted on the $\mathrm{x}$-axis and the average RMSE on the y-axis. The quality of the model increases with a decreasing distance to the origin. Note that we focus on the one week-ahead forecast since the results are similar for both forecast horizons but one week-ahead is slightly more difficult and computationally expensive.

Regarding the building with weak seasonal variation, we observe that the PNN models with a standard load profile are the closest to 


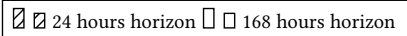

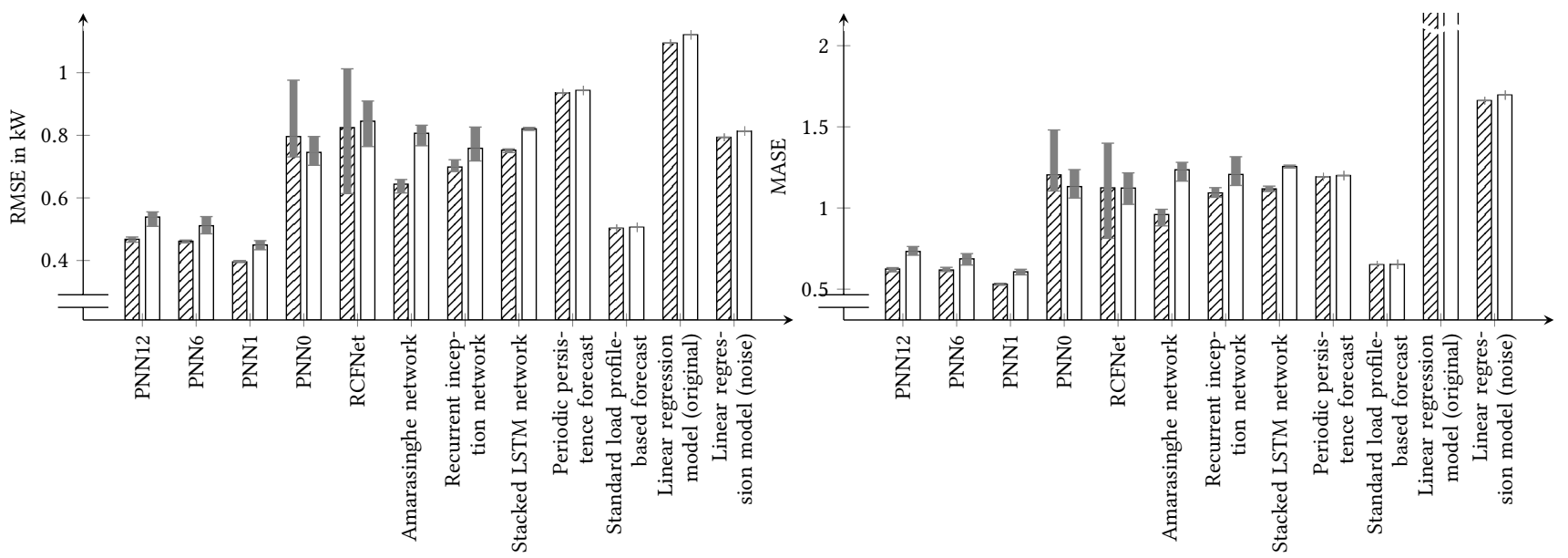

(a) The RMSE and the MASE of the PNN models and the benchmarks on the building with weak seasonal variation.

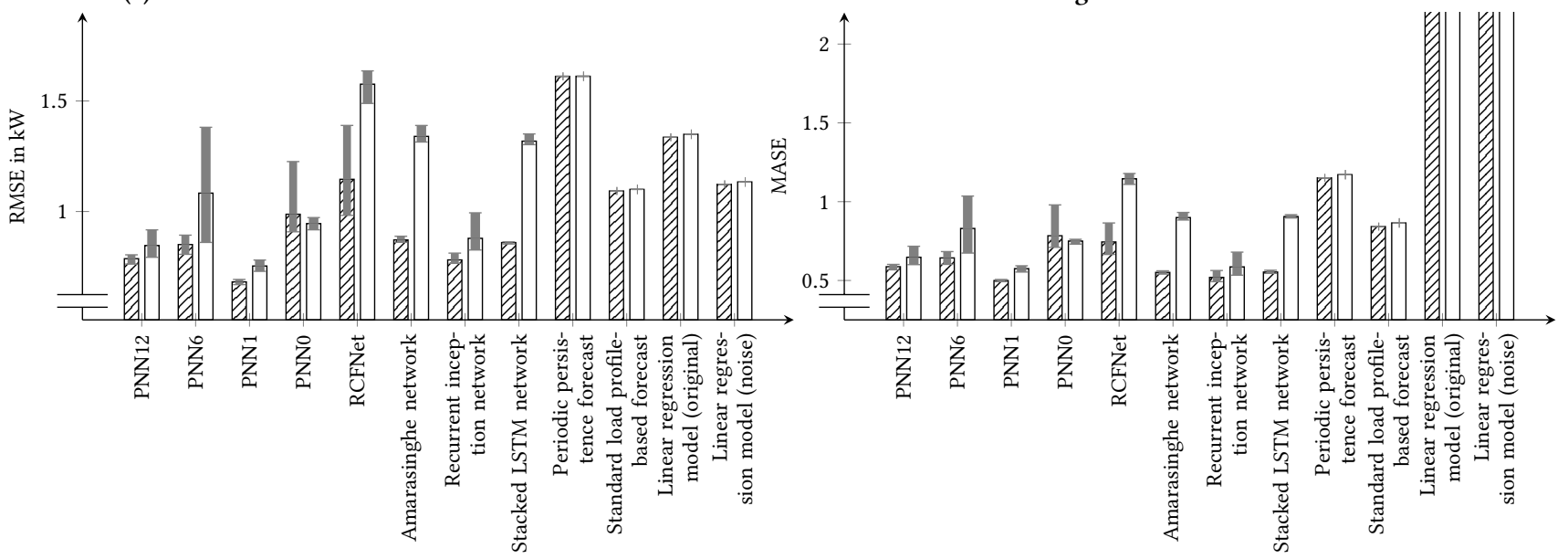

(b) The RMSE and the MASE of the PNN models and the benchmarks on the building with strong seasonal variation.

Figure 9: The RMSE and the MASE of the PNN models and the benchmark models on the selected buildings presented in Section 3.1. For each model, the bars indicates the average RMSE on the left side and the average MASE on the right side while the grey error bar shows the worst and the best observed RMSE and MASE respectively.

the origin. Moreover, three clusters and one outlier exist, i. e. the recurrent inception network that distinctly needs more training time than all other models. The three clusters comprise the RCFNet and the model without a standard load profile, the PNN models, and the Amarasinghe network together with the stacked LSTM network.

For the building with strong seasonal variation, PNN1 and PNN12 have the same distance to the origin. The PNN1 has the better accuracy while PNN12 needs less training time. In contrast to the scatter plot for the building with weak seasonal variation, clear clusters do not exist. However, the recurrent inception network is an outlier again.
Decomposition. With regard to the decomposition, we analyze one exemplary week. Figure 11 shows the decomposition of the electrical load into the standard load profile, the trend, and the colorful noise as well as prediction and ground truth for the week-ahead forecasts of PNN1 for December 21, 2015. On the fifth day of this week, i. e. 1st Christmas Holiday, the colorful noise improves the prediction by producing a highly negative value. Furthermore, we observe that the colorful noise and the trend mostly fluctuate around zero. This observation is confirmed by the weights assigned to the three modules. PNN1 assigns the highest weight to the standard load profile (0.96), followed by the trend (0.51) and the colorful noise (0.28). 
PNN12 PNN with 12 months profile PNN6 PNN with 6 months profile PNN1 PNN with 1 month profile PNN0 PNN without a profile RCF RCFNet AN Amarasinghe network RIN Recurrent inception network LN Stacked LSTM network

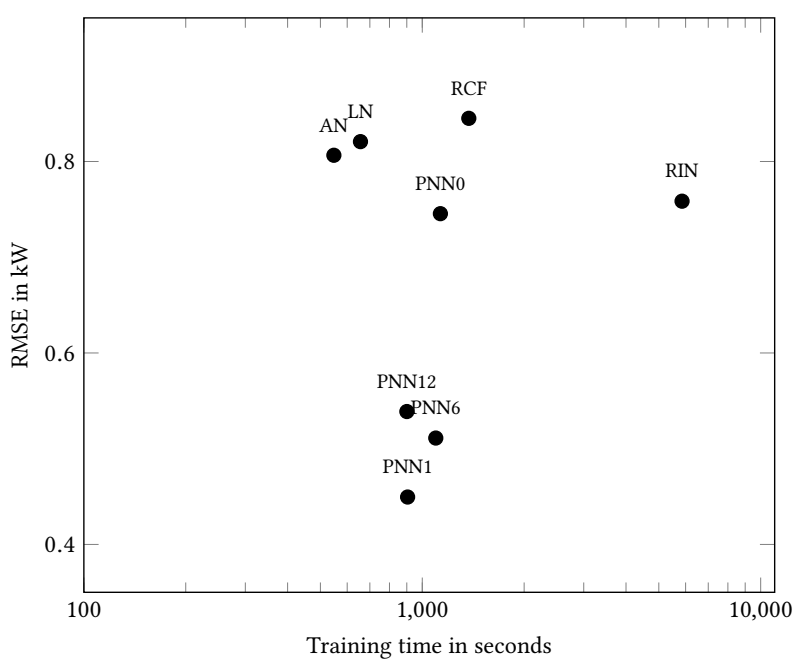

(a) The computational effort for the prediction of the building with weak seasonal variation.

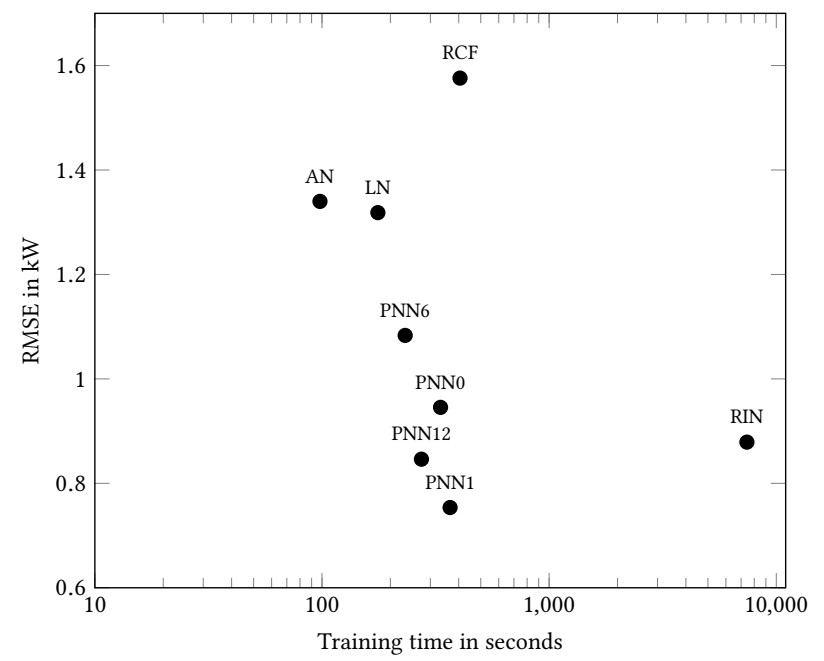

(b) The computational effort for the prediction of the building with strong seasonal variation.

Figure 10: Comparison of the average accuracy and the average training time needed of the PNN models and the benchmark models for one week-ahead forecast. The $\mathrm{x}$-axis indicates the the training time needed on a logarithmic scale and the $y$-axis the RMSE.

\section{DISCUSSION}

This section discusses the benefits of the architecture and the previously reported results regarding the accuracy, training time needed, potential trade-offs between accuracy and training time, and the decomposition of the electrical load.
Our proposed PNN model provides several benefits. The most important one is that the modules and encoders are exchangeable. Consequently, for different types of buildings, e. g. residential, office, or factories, one can use different modules and encoders. Moreover, our architecture allows detecting whether changes in the load refer to changes in the profile, trend, or the colorful noise. While the architecture is tested on building level consumption data, it was not specifically designed for this aggregation level and is thus independent of it. We assume that the architecture performs equally well on other aggregation levels, i. e. substation or country level.

Regarding the accuracy, we present four key observations. First, the results indicate that the PNN model provides better accuracy compared to the other architectures. Therefore, the decomposition of the time series and training of the decomposed parts with neural networks improves the forecast accuracy. Second, the comparison of the PNN models with and without a standard load profile shows that standard load profiles are suitable for supporting neural networks coping with periodicity. The accuracy of PNN0 is also distinctly worse than for example of PNN1. Third, small window sizes seem to improve the forecasting results, probably because smaller window sizes capture seasonal changes faster. And last, if strong seasonal variation is present in the time series, the window size should be selected carefully. As we can observe with the PNN6, some window sizes make the learning process for the network harder. In the 6 month window case, the profile reflects the seasonal influences with a delay of half a year. Therefore, the network has to offset this effect with the trend module and the colorful noise module, which depending on the initial weights can be difficult and could explain the large spread in accuracy.

Concerning the training time needed, we also discuss four observations. First, the PNN model with a standard load profile needs less training time than the PNN model without one. This observation indicates that the standard load profile supports the neural network during the training process. Consequently, the PNN model learns the load pattern more easily. Second, the recurrent inception network needs the longest training time. We assume that CNNs are more straightforward to parallelize than RNNs [4]. Furthermore, the PNN models significantly reduce the training complexity, as they, for example, need roughly 4,000 parameters to predict the next 168 hours, while the recurrent inception network needs more than 51,000 Third, all models, apart from the recurrent inception network for the one week-ahead forecast, need less training time for the building with strong seasonal variation. A possible explanation is that, for this building, the models mainly focus on the seasonality that is probably easier to learn than additional hidden knowledge. As a consequence, the models need less training time for the building with strong seasonal variation. Finally, all models, except the Amarasinghe network, need less training time for the one day-ahead forecast than the one week-ahead forecast. This result is in line with our expectation that the difficulty increases with the forecast horizon.

Considering both accuracy and training time needed, for both buildings and both forecast horizons, the PNN models are closest to the origin. This result indicates that while there are slightly faster models available, the additional training time is well invested as the error decreases significantly. 


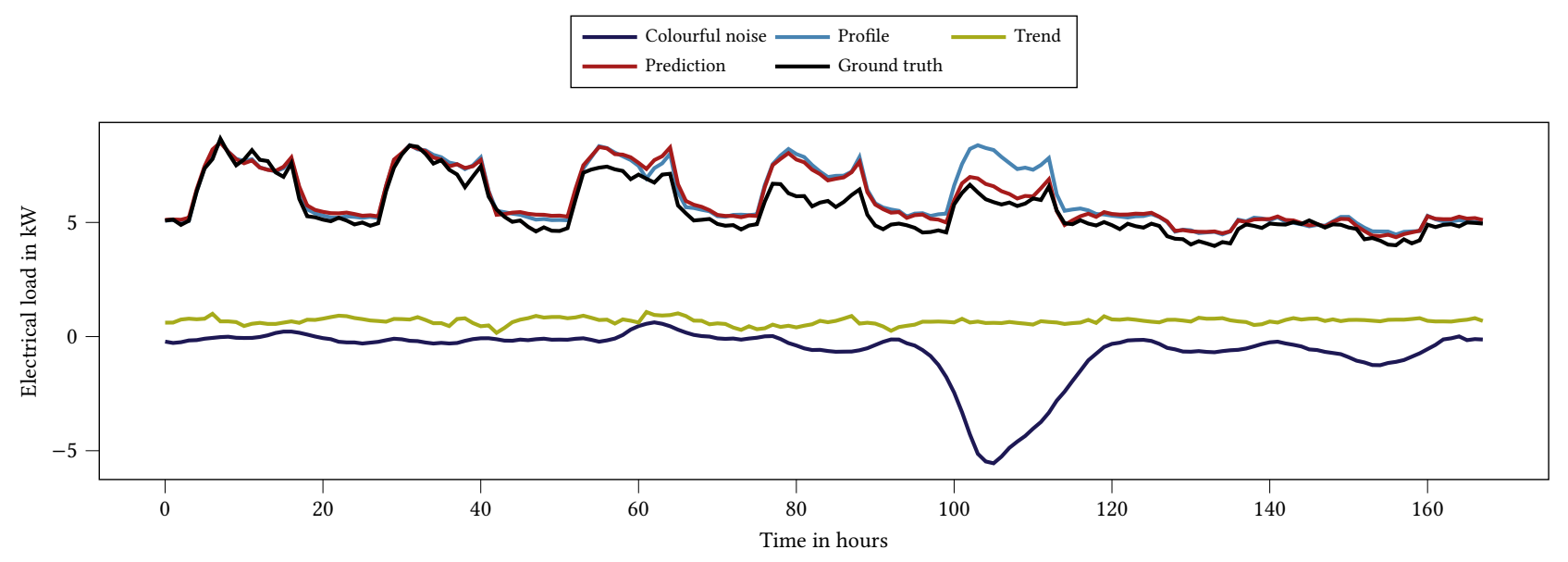

Figure 11: Decomposition of the time series as calculated by the PNN, together with the prediction and ground truth for the week starting at December 21, 2015.

Finally, regarding the decomposition, we observe that the trend and the colorful noise modules improve the prediction. In the mentioned example, the colorful noise module uses the information that December 25 is a public holiday. December 24, however, is not a public holiday and thus the corresponding load forecast is not corrected accordingly. This observation indicates that improved calender information could improve the forecasting accuracy. Moreover, since the values of both modules are mostly close to zero, we interpret both as a correction element. This interpretation is supported by the observation concerning the weights that the standard load profile is the most import module, followed by the trend and the colorful noise modules.

Overall, the results indicate that the combination of statistical information and neural networks offers advantages over both neural networks and methods based on statistical information only.

\section{CONCLUSION}

The present paper proposes a new deep neural network architecture for forecasting energy time series called profile neural network (PNN). Energy time series typically exhibit high periodicity, and in line with the finding that statistical information can improve deep learning, we give the fast and promising $\mathrm{CNN}$ explicit periodicity information in the form of a standard load profile. We apply the proposed deep neural network architecture to real-world electricity data from buildings on a university campus. For a building with weak seasonal variation and a building with strong seasonal variation, we evaluate the proposed architecture on two forecast horizons, namely one day and one week-ahead. Additionally, we compare the PNN model to four state-of-the-art deep learning models and three naive forecasting models regarding the accuracy, training time needed, and the trade-off between both.

The results show that the proposed PNN model has the best accuracy for both buildings, regardless of the magnitude of the seasonal variation. The introduction of standard load profiles thus supports neural networks coping with seasonal variation. If the seasonal variation present in the time series is strong, the window size used for the profile calculation has to be selected carefully, where smaller window sizes seem to perform better. Additionally, although the proposed model is slower than a simple $\mathrm{CNN}$ and similarly fast to the RCFNet, the additional training time is well invested when considering the substantial increase in forecast performance as well.

In future work, we want to further improve the architecture's performance by testing different profile calculation techniques, for example, considering seasonal standard load profiles and adding more weather information directly into the profile such as severe weather events. Additionally, we want to evaluate the PNNs performance on tasks such as concept drift and transfer learning and use publicly available data sets on different scales for that.

\section{ACKNOWLEDGMENTS}

The authors would like to thank Andreas Bartschat and Tillmann Mühlpfordt for their valuable feedback during the process and Kai Schmieder for providing the implementation of the Stacked LSTM network. Nicole Ludwig, Ralf Mikut, and Veit Hagenmeyer are supported by the Helmholtz Association under the Joint Initiative "Energy System 2050 - A Contribution of the Research Field Energy". Benedikt Heidrich is supported by Helmholtz AI for the research field energy.

\section{REFERENCES}

[1] Hesham K. Alfares and Mohammad Nazeeruddin. 2002. Electric load forecasting: Literature survey and classification of methods. International fournal of Systems Science 33, 1 (2002), 23-34. https://doi.org/10.1080/00207720110067421

[2] Abdulaziz Almalaq and George Edwards. 2017. A Review of Deep Learning Methods Applied on Load Forecasting. In 2017 16th IEEE International Conference on Machine Learning and Applications (ICMLA). IEEE, 511-516. https://doi.org/ 10.1109/ICMLA.2017.0-110

[3] Kasun Amarasinghe, Daniel L. Marino, and Milos Manic. 2017. Deep Neural Networks for Energy Load Forecasting Kasun. In 2017 IEEE 26th International Symposium on Industrial Electronics (ISIE). IEEE, 1483-1488. https://doi.org/10. 1109/ISIE.2017.8001465

[4] James Bradbury, Stephen Merity, Caiming Xiong, and Richard Socher. 2017. Quasi-Recurrent Neural Networks. In 5th International Conference on Learning Representations (ICLR 2017). OpenReview.net. https://openreview.net/forum?id= H1zJ-v5xl

[5] Mengmeng Cai, Manisa Pipattanasomporn, and Saifur Rahman. 2019. Dayahead building-level load forecasts using deep learning vs. traditional time-series 
techniques. Applied Energy 236 (2019), 1078-1088. https://doi.org/10.1016/j. apenergy.2018.12.042

[6] Chirag Deb, Fan Zhang, Junjing Yang, Siew Eang Lee, and Kwok Wei Shah. 2017. A review on time series forecasting techniques for building energy consumption. Renewable and Sustainable Energy Reviews 74 (2017), 902-924. https://doi.org/10. 1016/j.rser.2017.02.085

[7] DWD Climate Data Center (CDC). 2018. Historical Hourly Station Observations of $2 \mathrm{~m}$ Air Temperature and Humidity for Germany, Version V006. https: //cdc.dwd.de/portal/

[8] Carola Gerwig. 2015. Short Term Load Forecasting for Residential Buildings-An Extensive Literature Review. In Intelligent Decision Technologies, Rui NevesSilva, Lakhmi C. Jain, and Robert J. Howlett (Eds.). Springer, Cham, 181-193. https://doi.org/10.1007/978-3-319-19857-6

[9] Jorge Á. González Ordiano, Simon Waczowicz, Veit Hagenmeyer, and Ralf Mikut 2018. Energy forecasting tools and services. Wiley Interdisciplinary Reviews Data Mining and Knowledge Discovery 8, 2 (2018), e1235. https://doi.org/10.1002/ widm. 1235

[10] Ian Goodfellow, Yoshua Bengio, and Aaron Courville. 2016. Deep Learning. MIT Press.

[11] Sepp Hochreiter and Jürgen Schmidhuber. 1997. Long Short-Term Memory. Neural Computation 9, 8 (1997), 1735-1780. https://doi.org/10.1162/neco.1997.9. 8.1735

[12] Rob J. Hyndman and Anne B. Koehler. 2006. Another look at measures of forecast accuracy. International fournal of Forecasting 22, 4 (2006), 679-688. https://doi.org/10.1016/j.ijforecast.2006.03.001

[13] Junhong Kim, Jihoon Moon, Eenjun Hwang, and Pilsung Kang. 2019. Recurrent inception convolution neural network for multi short-term load forecasting. Energy and Buildings 194 (2019), 328-341. https://doi.org/10.1016/j.enbuild.2019. 04.034

[14] Diederik P. Kingma and Jimmy Lei Ba. 2015. Adam: A Method for Stochastic Optimization. In 3rd International Conference on Learning Representations (ICLR 2015). arXiv:1412.6980 http://arxiv.org/abs/1412.6980

[15] Ping-Huan Kuo and Chiou-Jye Huang. 2018. A High Precision Artificial Neural Networks Model for Short-Term Energy Load Forecasting. Energies 11, 1 (2018), 213. https://doi.org/10.3390/en11010213

[16] Yann LeCun, Yoshua Bengio, and Geoffrey Hinton. 2015. Deep learning. Nature 521 (2015), 436-444. https://doi.org/10.1038/nature14539

[17] Ming Lei, Liyang Tang, Mingxing Li, Zhenyu Ye, and Liwei Pan. 2019. Forecasting Short-Term Residential Electricity Consumption Using a Deep Fusion Model. In
Proceedings of 2018 Chinese Intelligent Systems Conference. Springer, Singapore, 359-371. https://doi.org/10.1007/978-981-13-2291-4_36

[18] T. Lonergan and J.V. Ringwood. 1995. Linguistic Modelling of Short-Timescale Electricity Consumption Using Fuzzy Modelling Techniques. In Irish DSP and Control Colloquium (IDSPCC 1995). http://mural.maynoothuniversity.ie/9531/1/ JR-Linguistic-1995.pdf

[19] Spyros Makridakis, Evangelos Spiliotis, and Vassilios Assimakopoulos. 2018. Statistical and Machine Learning forecasting methods: Concerns and ways forward. PLoS ONE 13, 3 (2018), e0194889. https://doi.org/10.1371/journal.pone.0194889

[20] Spyros Makridakis, Evangelos Spiliotis, and Vassilios Assimakopoulos. 2020. The M4 Competition: 100,000 time series and 61 forecasting methods. International fournal of Forecasting 36, 1 (2020), 54-74. https://doi.org/10.1016/j.ijforecast. 2019.04.014

[21] Daniel L. Marino, Kasun Amarasinghe, and Milos Manic. 2016. Building Energy Load Forecasting using Deep Neural Networks. In 42nd Annual Conference of the IEEE Industrial Electronics Society (IECON 2016). IEEE, 7046-7051. https: //doi.org/10.1109/IECON.2016.7793413

[22] Mohammad Azhar Mat Daut, Mohammad Yusri Hassan, Hayati Abdullah, Hasimah Abdul Rahman, Md Pauzi Abdullah, and Faridah Hussin. 2017. Building electrical energy consumption forecasting analysis using conventional and artificial intelligence methods: A review. Renewable and Sustainable Energy Reviews 70 (2017), 1108-1118. https://doi.org/10.1016/j.rser.2016.12.015

[23] K. Metaxiotis, A. Kagiannas, D. Askounis, and J. Psarras. 2003. Artificial intelligence in short term electric load forecasting: a state-of-the-art survey for the researcher. Energy Conversion and Management 44, 9 (2003), 1525-1534. https://doi.org/10.1016/S0196-8904(02)00148-6

[24] J. V. Ringwood, D. Bofelli, and F. T. Murray. 2001. Forecasting Electricity Demand on Short, Medium and Long Time Scales Using Neural Networks. Fournal of Intelligent and Robotic Systems 31, 1-3 (2001), 129-147. https://doi.org/10.1023/A: 1012046824237

[25] Slawek Smyl. 2020. A hybrid method of exponential smoothing and recurrent neural networks for time series forecasting. International fournal of Forecasting 36, 1 (2020), 75-85. https://doi.org/10.1016/j.ijforecast.2019.03.017

[26] Christian Szegedy, Wei Liu, Yangqing Jia, Pierre Sermanet, Scott Reed, Dragomir Anguelov, Dumitru Erhan, Vincent Vanhoucke, and Andrew Rabinovich. 2015. Going Deeper with Convolutions. In 2015 IEEE Conference on Computer Vision and Pattern Recognition (CVPR). IEEE, 1-9. https://doi.org/10.1109/CVPR.2015. 7298594 